\title{
The Inelastic Mean Free Path of Electrons. Research in Budapest, Warsaw, Wrocław and Clermont-Ferrand. Brief History and New Results
}

\author{
G. Gergely ${ }^{a, *}$, S. Gurban ${ }^{a}$, M. Menyhard $^{a}$, A. Jablonski $^{b}$ \\ AND L. ZOMMER ${ }^{b}$ \\ ${ }^{a}$ Research Institute for Technical Physics and Materials Science \\ P.O. Box 49, H-1525 Budapest, Hungary \\ ${ }^{b}$ Institute of Physical Chemistry, Polish Academy of Sciences \\ Kasprzaka 44/52, 01-224 Warsaw, Poland
}

The inelastic mean free path of electrons (IMFP) is an important material parameter for description of electron transport processes in solids. This parameter is particularly useful for quantifying the electron spectroscopies, in particular Auger electron spectroscopy, X-ray photoelectron spectroscopy, electron energy loss spectroscopy and elastic peak electron spectroscopy. In this work, a brief overview of the IMFP determination is presented. Generally, there are two groups of methods to determine the IMFP: (i) calculations using the theoretical model based on the experimental optical data, and (ii) calculations using theory relating the IMFP and the measured probability elastic electron backscattering from solids. Major advances in the development of the second group of methods were made in three laboratories; these advances are reviewed here. The elastic backscattering probability, in absolute or relative units, can be conveniently evaluated from the elastic peak intensity. However, much effort is needed to develop the theory for calculating the IMFP, which typically involves the Monte Carlo simulations of electron trajectories in solids. Presently, this theory and typical procedures of the spectra processing are implemented in the software package EPESWIN developed by Jablonski. In recent years, much attention is devoted to the phenomenon of the electron energy losses in the surface region of solids. Reliability of the theory of elastic backscattering is distinctly improved if this effect is taken into account.

PACS numbers: 81.05.Cy, 82.80.Dx, 82.80.Pv

*corresponding author; e-mail: gergely@mfa.kfki.hu 


\section{Introduction}

The inelastic mean free path of electrons (IMFP) $\lambda_{\mathrm{i}}$ is a fundamental material parameter for surface science, physics and quantitative analysis of surfaces and thin films. In addition to the scattering cross-sections (elastic and inelastic), the IMFP is needed to describe the electron transport processes, the $N(E)$ backscattering spectra, the Auger (AES) and X-ray photoelectron spectroscopy (XPS) peaks, the loss spectrum (REELS) and the elastic peak $I_{\mathrm{e}}$. For surface analysis, the IMFP $\lambda_{\mathrm{i}}$ is related to the information depth. The published theoretical models for determining the IMFP have been compiled and discussed by Powell and Jablonski [1].

Our paper at first presents a brief survey of the history of IMFP. This is followed by the summary of the theoretical [1] and experimental methods [2]. Finally, we describe our recent results.

The concept of the IMFP has been introduced by Clausius (1822-88), in the kinetic theory of gases. The "electron mean free path term" was used in the historical book of Seitz (Modern Theory of Solids, 1940). For semiconductors, the classical book of Shockley (Electrons and Holes in Semiconductors, 1950) deals with IMFP.

The $\lambda_{\mathrm{i}}$ parameter is defined in the ASTM (E-42, Comm. Standards 673, $1997)$ and ISO (18115/5.168:2001) standards. The IMFP is: "the average of distances, measured along trajectories, that a particle with a given energy travels between inelastic collisions in a substance". The IMFP is a parameter different from the escape depth or the attenuation length $\left(\lambda_{\mathrm{AL}}\right)$ of electrons [3].

\section{Calculation of the IMFP}

Several methods have been developed. They were summarized by Jablonski and Powell [1], where also detailed citations were given. Reasonable estimates were given in 1962 by Quinn. Powell started in 1974, pointing the use of experimental energy loss function or optical data for calculating the IMFP. Penn joined in 1974. From 1979 till 1988 Ashley et al. calculated the IMFP for many elements, using the Linhard dielectric function. After many approaches in the year 1988 Tanuma, Powell and Penn published the IMFP for 56 materials, elements and compounds, by applying the Penn algorithm. Their TPP-2M model was published in 1994 [4]. Nowadays this model and the NIST database SRD 71 are generally used for surface analysis, evaluation of experiments by Monte Carlo (MC) simulation of electron transport processes. The calculated IMFP data are summarized in [1]. Powell et al. continued research on the IMFP. Hereupon his latest work should be referred [5]. 


\section{Experimental determination of the IMFP}

\subsection{The overlayer method}

Experimental determination of the attenuation length applied the overlayer method [1, 3]. Thin overlayers are deposited in vacuum and the attenuation of photoelectrons, XPS or AES peaks are measured. This method requires: perfect, uniform and pinhole free very thin overlayers. Heteroepitaxy, Frank-van der Merve type growth is confined to certain overlayer/substrate materials. To avoid exposure to the atmosphere, in situ preparation is needed. This is possible in molecular beam epitaxy systems (MBS). Ar ${ }^{+}$ion bombardment cleaning produces artifacts. Magnetron plasma is now a useful technology. Energy losses on the interfaces cannot be avoided. Seah-Dench SIA [3] summarized the $\lambda_{\mathrm{AL}}$ data available in 1979.

\section{2. $E P E S$}

In the year 1981 the elastic peak electron spectroscopy (EPES) was introduced. A review is given in [2]. Now EPES is defined by ISO standards (ISO 18115/7.26:2001/PDAM2). The elastic (or rather quasielastic) [2] reflection coefficient $r_{\mathrm{e}}$ of electrons is composed by electrons escaping within $2 \pi$ solid angle. For any type of electron spectrometer, the parameter $r_{\mathrm{e}}(E, Z, \Omega)$ is derived from the elastic peak, within the spectrometer solid angle $\Omega$. The intensity $I_{\mathrm{e}}$ is determined by the primary energy $E_{\mathrm{p}}$, the atomic number $Z$, the IMFP and by the angle of incidence $\alpha_{\mathrm{i}}$ and of detection $\alpha_{\mathrm{d}}$. It is now a well known procedure to determine $\lambda_{\mathrm{i}}$ from the elastic peak intensity. This was started by Jablonski et al. in 1984 [6], and the research was continued permanently. EPES proved to be a practical tool for experimental determination of the IMFP. It is suitable for any solid surface, atomically smooth, and free from contamination. It is presently recommended for determination of the IMFP in agreement with ISO definition [1]. Several types of electron spectrometers were used in the past.

The retarding field analyzer (RFA) is operated with large $\Omega, 5-56^{\circ}$, and $E=20-2000 \mathrm{eV}$ energy range. The problems are: poor energy resolution $\Delta E_{\mathrm{s}}$, the transmission $T_{\mathrm{r}}(E)$ and response $R(E)$ are problematic. This difficulty was eliminated by Mróz et al. [7] by biasing the sample and deflecting the electron beam to the detector, $r_{\mathrm{e}}(E)_{\mathrm{RFA}}=i_{\mathrm{e}}(E) / i_{\mathrm{p}}$. Later, Bideux elaborated the optimum biasing conditions [8]. Mróz et al. [9] studied noble metals and Bondot evaluated the RFA experiments by applying his MC procedure [9]. The IMFP-EPES experimental results are summarized in $[1,2]$.

The better $\Delta E_{\mathrm{s}}$ and reasonable large $\Omega$ detector angular window of the cylindrical mirror analyzer (CMA) proved to be practical. CMA experiments were started in Budapest [6], composing the elastic peak intensity ratio $I_{\mathrm{e}, \text { sample }} / I_{\mathrm{e} \text {,ref }}$ for the sample and selected reference materials like $\mathrm{Al}, \mathrm{Ni}, \mathrm{Cu}$, Si, etc., thus the problems of $T_{\mathrm{r}}(E)$ and $R(E)$ were eliminated. The IMFP of the reference sample applied the NIST calculated TPP-2M values. MC simulation of the CMA experiments was elaborated by Jablonski et al. [10]. 
Standard samples $\mathrm{Ni}, \mathrm{Cu}$ were elaborated [2]. Problems of the CMA experiments are: the poor but better than RFA $\Delta E_{\mathrm{s}}$, produced large background in the vicinity of $E_{\mathrm{p}}$ and thus integrated also the loss spectrum. Procedures have been developed for background correction in EPES [2]. These difficulties were successfully eliminated since 1995 by making the EPES experiments with the hemispherical analyzer (HSA) ESA-31, developed by ATOMKI-Debrecen [2].

IMFP-EPES experiments supply the experimental IMFP denoted by $\lambda_{\mathrm{e}}$. Our paper is confined to IMFP-EPES experiments, carried out in Budapest (CMA), Warsaw (double-pass CMA), Clermont-Ferrand (RFA and HSA), Lyon (HSA), Debrecen (ESA-31), Odense (HSA) and Prague (RFA). Results on metals, semiconductors, polymers [11] are summarized in [2].

\subsection{AREPES}

Angular resolved EPES (AREPES) has been developed in Tuebingen, Eberhard-Karl's University. Several thesis works applied their special angular spectrometer [2]. They published IMFP results on several metals. Their $\mathrm{d} r_{\mathrm{e}}\left(E, \alpha_{\mathrm{d}}\right) / \mathrm{d} \Omega$ AREPES spectra are presented in figures in absolute units. Results are summarized in [2].

\subsection{MC calculation}

Evaluation of EPES-IMFP experiments with the use of the MC simulation, was started by Jablonski in 1985 [1]. He achieved further development of MC for EPES in 1985-2005. Nowadays the most advanced version and software EPESWIN [12] of Jablonski is generally used. It is based on the NIST SRD 64 Elastic Scattering Cross-Sections and on the NIST SRD 71 IMFP databases.

\section{Surface excitation losses}

The main problem with IMFP-EPES is $\lambda_{\mathrm{e}}<\lambda_{\mathrm{i}}$. In 1994, Chen et al compared experimental and calculated EPES spectra $[2,13]$. They observed that below $200 \mathrm{eV}, I_{\mathrm{e}, \exp }<I_{\mathrm{e}, \text { calc }}$. They explained the discrepancy by surface excitation losses. Systematic research started in 1998. Kwei et al. introduced the surface excitation material parameter (SEP) [14]. In 2000 Tanuma et al. [15] assumed Poissonian surface excitation process and they defined an exponential $f_{\mathrm{s}}$ correction factor for the experimental elastic peak intensity: $I_{\mathrm{e}} f_{\mathrm{s}}=I_{\mathrm{c}}$ (calculated). $f_{\mathrm{s}}=1 / \exp \left(-P_{\mathrm{se}}\right)$ with $P_{\mathrm{se}} \mathrm{SEP}$ parameter. Now this is defined by ISO (18115/7.80:2001/PDAM2). Models have been elaborated by Oswald [16] and Chen [17]. The most recent results and material parameters are published by Werner et al. [18], Nagatomi and Goto [19], Kwei et al. [20] and Gergely et al. [21].

The $P_{\text {se }}=P_{\text {sei }}+P_{\text {seo }}$, is produced by incident (i) and escaping (o) electrons. They can be described by two different types of relationship. The Oswald-Werner $[16,18]$ model gives 


$$
P_{\mathrm{sei}}=\frac{1}{0.171 a_{\mathrm{W}} \sqrt{E} \cos \alpha_{\mathrm{i}}+1} .
$$

$a_{\mathrm{W}}$ is a material parameter, determined recently by Werner [18]. $\alpha_{\mathrm{i}}$ is the angle of incidence. Similar equation is valid for escaping electrons.

The other relationship was introduced by Kwei et al. [20]. Its more recent version $[20]$ is

$$
P_{\text {sei }}=a E^{-b} / \cos ^{c} \alpha_{\mathrm{i}} .
$$

$a, b$ and $c$ are material parameters. Chen [17] worked with $a_{\text {ch }}$ material parameters, $b=0.5, c=1$. Kwei et al. [20] used 6 parameters. Some authors determined the material parameters by best fitting of experimental results. They will be compared in Sect. 5.

\section{Experimental determination of the SEP parameter}

Four kinds of experimental methods have been used by several authors. In our laboratory, like for the IMFP-EPES experiments, the ratio $I_{\mathrm{e}, \mathrm{sample}} / I_{\mathrm{e}, \text { ref }}$ was subjected to SEP correction [21] with Tanuma's relationship [15]:

$$
I_{\mathrm{es}} / I_{\mathrm{er}}=I_{\mathrm{cs}} \exp \left(-P_{\mathrm{ser}}\right) / I_{\mathrm{cr}} \exp \left(-P_{\mathrm{ses}}\right)
$$

with notations: $I_{\mathrm{es}}$ experimental, $I_{\mathrm{cs}}$ calculated elastic peak intensity and similarly for $I_{\mathrm{er}}, I_{\mathrm{cr}}, P_{\mathrm{ses}}$ and $P_{\mathrm{ser}}$, respectively. The experimental $I_{\mathrm{e}}$ intensities are in arbitrary units, only their ratio is needed. The $I_{\mathrm{c}}$ data were calculated for the spectrometer angular conditions with MC EPESWIN [12] algorithm. The $\lambda_{\mathrm{i}}$ IMFPs were TPP-2M data. For the reference, $P_{\text {ser }}$ was taken from Chen [17] and modified for better approach [21,22]. This method might be useful, provided that reliable $P_{\text {ser }}$ data are available. It is practical to work with a spectrometer of good $\Delta E_{\mathrm{s}}$ (e.g. HSA).

Several attempts have been made with highly resolved REELS spectra. The recent works of Pauly et al. [23] and Werner et al. [18] apply Eq. (1) of Oswald [16] and they published experimental $a_{\mathrm{W}}$ material parameters. These authors simulate the experimental REELS spectra of high $\Delta E_{\mathrm{s}}$ resolution, but in arbitrary units. The SEP parameter is deduced from the distribution of the REELS spectrum. Very recently Nagatomi and Goto [19] analyzed Goto's experimental spectra [24] on $\mathrm{Ni}$, presented in absolute units. They determined $a_{\mathrm{chNi}}=4.3$ and the IMFPs of $\mathrm{Ni}$ as well. Very good agreement was achieved with the TPP-2M data.

Our paper is confined to Si. In our recent work we deduced the SEP parameters from Goto's elastic current data in absolute units. Tanuma's exponential relationship was applied [15]. The SEP parameters were deduced for $\mathrm{Si}, \mathrm{Ni}, \mathrm{Cu}$, $\mathrm{Ag}$ and $\mathrm{Au}$, using both relationships in Eq. (1) and Eq. (2). For Chen $a_{\mathrm{chSi}}$ was determined by best fitting. According to Eq. (4) the minimum of $\Delta I_{\mathrm{e}}$ was searched by trial and error for determination of $a_{\mathrm{chSi}}$ :

$$
\Delta I_{\mathrm{e}}[\%]=100 \frac{1}{n} \sum_{i=1}^{n}\left|\frac{I_{\mathrm{e}}-I_{\mathrm{ec}}}{I_{\mathrm{ec}}}\right| .
$$


The experimental $I_{\mathrm{e}}(E)$ is uncorrected for surface excitation. The SEP corrected $f_{\mathrm{s}}(E) I_{\mathrm{e}}(E)$ curves were calculated for the material parameters published on Si. They are summarized in Table. Results are presented in Fig. 1 which compares the experimental $I_{\mathrm{e}}(E)$, and the SEP corrected $f_{\mathrm{s}} I_{\mathrm{e}}(E)$ with the $I_{\mathrm{c}}(E)$ calculated backscattered elastic intensities (using the $\lambda_{\mathrm{i}}$ TPP-2M data as input parameters).

Recently Jablonski and Zemek [25] applied AREPES for determining the SEP material parameters using Eq. (1) and Eq. (2). They analyzed the experimental and calculated angular distribution of the elastic peak by normalizing the experimental and calculated (with and without SEP correction) curves. Their $a_{\mathrm{ch}}$ and $a_{\mathrm{W}}$ material parameters are included in Fig. 1 and Table.

TABLE

The material parameters applied for SEP correction for Si, by different authors and models.

\begin{tabular}{|c|c|c|c|c|c|c|}
\hline Author & Method & $\Delta \lambda_{\mathrm{coC}}[\%]$ & $\begin{array}{c}a_{\mathrm{ch}} \\
\text { in Eq. (2) }\end{array}$ & $\begin{array}{c}a_{\mathrm{W}} \\
\text { in Eq. (1) }\end{array}$ & $\Delta \lambda_{\mathrm{coO}}[\%]$ & Ref. \\
\hline Chen & calculated & 7.95 & $\begin{array}{c}2.5 \\
\text { (original) }\end{array}$ & & & {$[17]$} \\
\hline Werner & REELS & & & 1.2 & 3.7 & [18] \\
\hline $\begin{array}{c}\text { Jablonski- } \\
\text { Zemek }\end{array}$ & AREPES & 6.45 & 2.996 & 0.724 & 24.1 & {$[25]$} \\
\hline Pauly & REELS & & & 0.67 & & {$[23]$} \\
\hline $\begin{array}{c}\text { Present } \\
\text { work }\end{array}$ & EPES & 3.9 & 3.5 & 1.2 & 3.7 & \\
\hline Kwei et al. & AREPES & 11.2 & \multicolumn{3}{|c|}{$\begin{array}{c}a=1.1313, b=0.4756 \\
c=1.12 \text { (incidence) } \\
a=1.7295, b=0.4201 \\
c=0.83 / 16 \text { (escape) }\end{array}$} & {$[20]$} \\
\hline
\end{tabular}

The deviation $\Delta \lambda_{\text {co }}$ between $\lambda_{\text {co }}$ (SEP corrected) and $\lambda_{\mathrm{i}}$ (TPP-2M) data are included in Table. The average deviations between $\lambda_{\mathrm{i}}$ and $\lambda_{\text {co }}$ are deduced from EPES experiments and calculated with Eq. (4). They are denoted by $\Delta \lambda_{\mathrm{CoC}}$ for modified Chen using Eq. (2). $\Delta \lambda_{\mathrm{coO}}$ is the same with Eq. (1) (Oswald). $\Delta \lambda_{\mathrm{u}}$ for the uncorrected IMFP is $25 \%$.

The $I_{\mathrm{e}}(E)$ experimental spectra were taken from Goto's database [24]. The uncorrected and SEP corrected $\lambda_{\text {co }}$ IMFPs have been deduced from $I_{\mathrm{e}}(E)$ and $f_{\mathrm{s}} I_{\mathrm{e}}(E)$ data by applying the EPESWIN software. They are presented in Fig. 2. The $P_{\text {se }}$ curves deduced from experiments and used for the $f_{\mathrm{s}}$ parameters are shown in Fig. 3. The experimental $P_{\mathrm{se}}=\ln \left(I_{\mathrm{c}} / I_{\mathrm{e}}\right)$. 


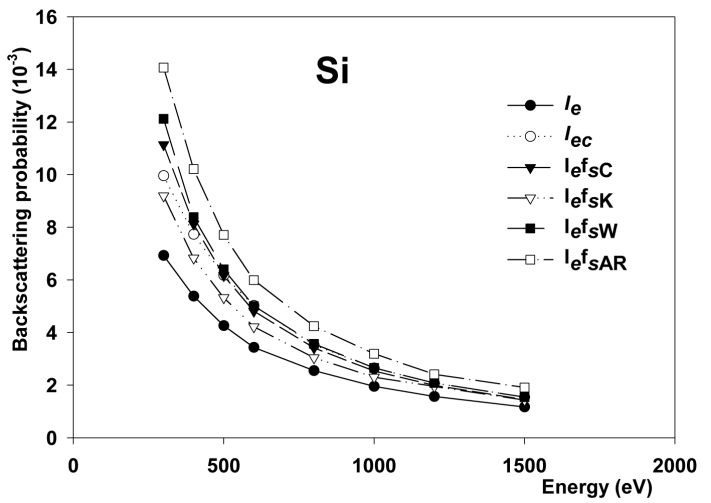

Fig. 1. Comparison of the calculated $I_{\mathrm{c}}(E)$, the uncorrected $I_{\mathrm{e}}$ and the SEP corrected $f_{\mathrm{s}} I_{\mathrm{e}}$ curves. $f_{\mathrm{sAR}}$ is the SEP correction factor using the material parameter of AREPES (Oswald) [20].

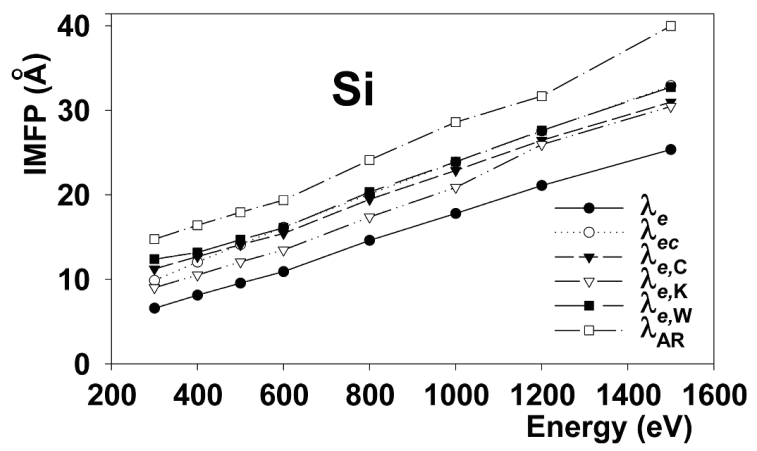

Fig. 2. Comparison of the uncorrected and SEP corrected IMFPs $\lambda_{\text {co }}$ with the TPP-2M data.

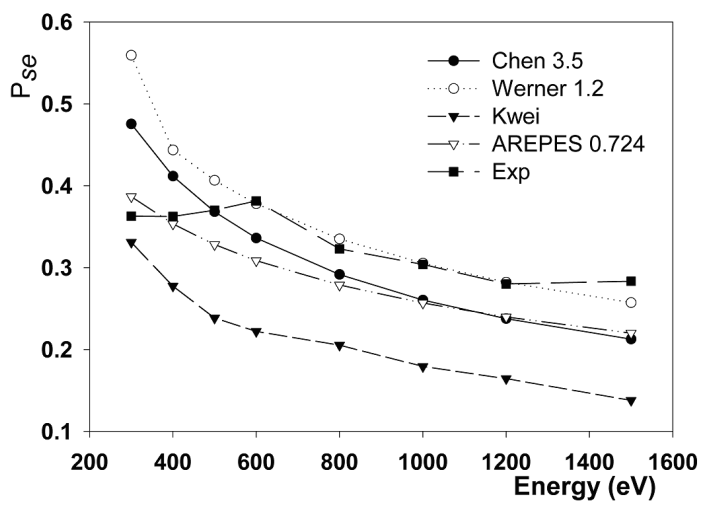

Fig. 3. Comparison of the $P_{\text {se }}$ SEP parameters deduced from experiments and used for calculating the $f_{\mathrm{s}}$ correction factors. In the figure exp denotes $f_{\mathrm{s}}$ deduced from $\ln \left(I_{\mathrm{c}} / I_{\mathrm{e}}\right)$. 


\section{Quantification applying the Goto database}

In general, electron spectra (AES, XPS, REELS, and EPES) and the peaks as well are presented in arbitrary units. In quantitative surface analysis, the peaks of reference samples are compared with the Auger or XPS peak heights. Our goal is the determination of the elastic peak $I_{\mathrm{e}}(E)$ and of the backscattering spectra $N(E)$ in absolute units. Nagatomi and Goto [19] determined the IMFP and SEP parameter of Ni applying Goto's database [24] in absolute units. Experimental determination of the SEP parameters needs also $I_{\mathrm{e}}(E)$ in absolute units. In our present work, quantification of EPES and SEP correction applied also Goto's database. It presents the current $i(E)$ of the backscattering spectra, measured with special CMA for $E$ energies and $E_{\mathrm{p}}$ primary energies on a great number of elements, for $i_{\mathrm{e}}=1 \mu \mathrm{A}$ primary current. Recently, Goto et al. published the $T_{\mathrm{r}}(E)$ transmission of his CMA [26]. Thus, the absolute values of elastic and inelastic backscattering $N(E)$ spectra can be deduced. In our present work, the integrals of $N(E)$ spectra with boundaries $50 \mathrm{eV}$ and $E_{\mathrm{p}}$ were also used. They supply the backscattering yield (BY) (ISO18115/5.96:2001) for the CMA angular window. Zommer et al. calculated BY for $2 \pi$ and angular correction $f\left(\alpha_{\mathrm{d}}\right)$, too, for the CMA [27]. A brief review of our procedure is given in the following steps. The $i\left(E, E_{\mathrm{p}}\right)$ current measured by Goto in $\mathrm{nA}$ units is detected with $\Delta E_{\mathrm{s}}=0.0025 E \mathrm{eV}$ units. In the first step it is converted to $\Delta E_{\mathrm{s}}=1 \mathrm{eV}$ resolution. This approach is valid for the slowly varying continuous spectrum, but not valid for the fine structure of the loss spectrum and neither for the elastic peak. In the second step it is corrected by $T_{\mathrm{r}}(E)$ transmission [26]. The probability of backscattering an electron for $E$ and within $\Delta E_{\mathrm{s}}=1 \mathrm{eV}$ is denoted by $N(E) . N(E)$ in absolute units will be

$$
N(E)=i(E) \times 10^{-6} /\left(0.0025 E T_{\mathrm{r}}(E)\right) .
$$

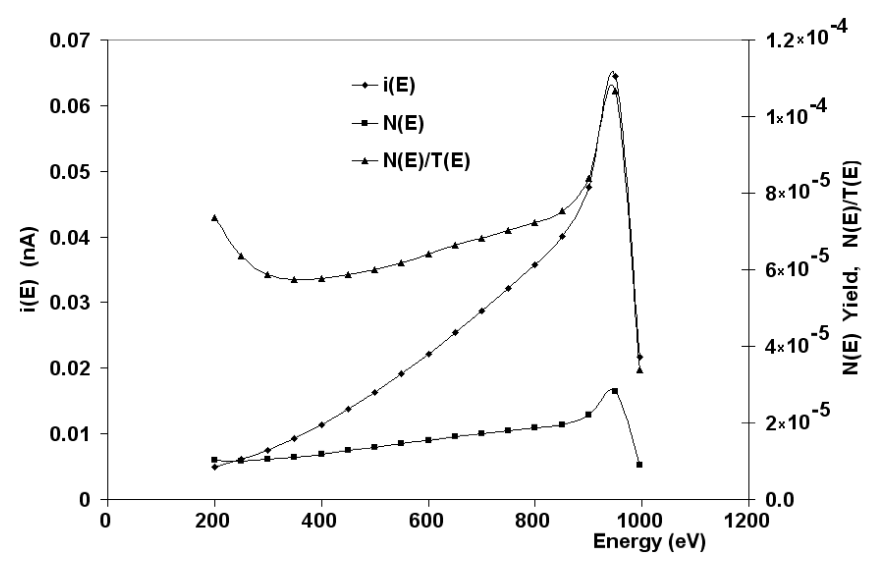

Fig. 4. Transformation of Goto's $i(E)$ data to $N(E)$ for $E_{\mathrm{p}}=1 \mathrm{keV}, \Delta E_{\mathrm{s}}=1 \mathrm{eV}$. 
$N(E)$ is the number of backscattered electrons/incidents detected by the CMA for $i_{\mathrm{p}}=1 \mu \mathrm{A}, \Delta E_{\mathrm{s}}=1 \mathrm{eV}$. In Fig. $4, i(E)$ and $N(E)$ are presented on $\mathrm{Si}$ for $E_{\mathrm{p}}=1 \mathrm{keV}, \alpha_{\mathrm{i}}=0^{\circ}, \alpha_{\mathrm{d}}=42.3 \pm 6^{\circ}$.

Figure 4 is valid for Goto's CMA and $1 \mathrm{keV}$. In our present work, it is assumed that the distribution of $N(E)$ is similar for smaller angular windows, e.g. a half cone of $\alpha=4^{\circ}$ or $6^{\circ}$. The detected signal is proportional to its solid angle. This was verified by our MC calculations. The BY corrected for $\alpha_{\mathrm{d}}$ is

$$
\int_{50}^{E_{\mathrm{p}}} N(E) \mathrm{d} E=\operatorname{BY}(E) f\left(\alpha_{\mathrm{d}}\right) .
$$

The $T_{\mathrm{r}}(E)$ of the CMA for $E<400 \mathrm{eV}$ strongly decreases, therefore the integral was confined and corrected to $200 \mathrm{eV}$ boundaries. The other problem is the superposition of secondary and of backscattered electrons in the $E=$ 50-200 eV range. Equation (4) offers a possibility for quantifying $N(E)$ spectra for any electron spectrometer, e.g. HSA, and its calibration as well. For this purpose, the $N(E)$ spectra, valid for Goto's CMA are to be transformed. This needs further research.

\section{Conclusions}

The IMFP determined by any kind of experiments is uncorrected for surface excitation losses. The brief review of the history of IMFP research was confined to those. Except the method of $I_{\mathrm{e}, \text { sample }} / I_{\mathrm{e}, \text { ref }}$, the $R(E)$ spectrometer response is a crucial problem. The $\Delta E_{\mathrm{s}}$ energy resolution should be taken into consideration and background correction cannot be avoided.

The goal of present research is the surface excitation, its models and material parameters. Perfect solution was achieved for Ni only by Nagatomi, evaluating REELS spectra in absolute units. Reasonable solution was found for $\mathrm{Si}$ (low $Z$ element), as observed on SEP corrected $I_{\mathrm{e}}(E)$ and IMFP curves. Best SEP correction was achieved with $a_{\mathrm{W}}=1.2$ of Werner [18].

\section{Acknowledgments}

Authors highly appreciate the scientific works of Professor Stefan Mróz and express their best wishes.

Many results were presented at the Seminars of Surface Physics, organized by the Institute of Experimental Physics of the University of Wrocław.

Our most recent results were achieved within the Cooperation Project PL-15 of the Polish and Hungarian Academy of Sciences. The brief history of the IMFP was reported about results of the EU COPERNICUS project, the cooperation projects TET-Balaton, and MTA-CNRS (Budapest-Warsaw-Clermont-Ferrand). Authors appreciate their successful cooperation with the Partners. 


\section{References}

[1] C.J. Powell, A. Jablonski, J. Phys. Chem. Ref. Data 28, 19 (1999).

[2] G. Gergely, Prog. Surf. Sci. 71, 31 (2002).

[3] M. Seah, W.A. Dench, Surf. Interface Anal. 1, 2 (1979).

[4] S. Tanuma, C.J. Powell, D.R. Penn, Surf. Interface Anal. 21, 165 (1994).

[5] C.J. Powell, A. Jablonski, F. Salvat, S. Tanuma, D.R. Penn, J. Surf. Anal. 12, 88 (2005).

[6] A. Jablonski, P. Mrozek, G. Gergely, M. Menyhard, A. Sulyok, Surf. Interface Anal. 6, 291 (1984).

[7] W. Dolinski, H. Nowicki, S. Mróz, Surf. Interface Anal. 11, 229 (1988).

[8] L. Bideux, Ph.D. Thesis, No. 609, Univ. Blaise-Pascal, Clermont-Ferrand 1994.

[9] W. Dolinski, S. Mróz, J. Palczynski, B. Gruzza, P. Bondot, A. Porte, Acta Phys. Pol. A 81, 193 (1992).

[10] A. Jablonski, B. Lesiak, G. Gergely, Phys. Scr. 39, 363 (1989); Vacuum 40, 67 (1990).

[11] B. Lesiak, A. Kosinski, A. Jablonski, A. Sulyok, G. Gergely, J. Tóth, D. Varga, Acta Phys. Pol. A 109, 789 (2006).

[12] A. Jablonski, Surf. Interface Anal. 37, 1034 (2005).

[13] Y.F. Chen, P. Su, C.M. Kwei, C.J. Tung, Phys. Rev. B 47, 17547 (1994).

[14] C.M. Kwei, C.Y. Wang, C.J. Tung, Surf. Interface Anal. 26, 682 (1998).

[15] S. Tanuma, K. Ichimura, K. Goto, Surf. Interface Anal. 30, 212 (2000).

[16] R. Oswald, Ph.D. Thesis, Eberhard-Karls Univ. Tuebingen, 1992.

[17] Y.F. Chen, Surf. Sci. 519, 115 (2002).

[18] W.S.M. Werner, L. Kover, S. Egri, J. Toth, D. Varga, Surf. Sci. 585, 85 (2005).

[19] T. Nagatomi, K. Goto, Appl. Phys. Lett. 87, 224107 (2005); Phys. Rev. B 75, 235424 (2007).

[20] C.M. Kwei, Y.C. Li, C.J. Tung, Surf. Sci. 600, 3690 (2006).

[21] G. Gergely, M. Menyhard, S. Gurban, J. Toth, D. Varga, A. Jablonski, J. Surf. Anal. 12, 140 (2005).

[22] G. Gergely, M. Menyhard, S. Gurban, J. Toth, D. Varga, Surf. Interface Anal. 36, 1098 (2004).

[23] M. Pauly, S. Tougaard, F. Yubero, Surf. Interface Anal. 37, 1151 (2005).

[24] K. Goto, http://www.sasj.jp/compro.

[25] A. Jablonski, J. Zemek, Surf. Sci. 601, 3409 (2007).

[26] Adel Alkafri, Y. Ichikawa, R. Shimizu, K. Goto, J. Surf. Anal. 14, 2 (2006).

[27] L. Zommer, A. Jablonski, G. Gergely, S. Gurban, Vacuum 82, 201 (2008). 